where the $L_{i}$ are nonsingular square matrices and where $L_{i}$ is the transpose of $L_{m-i}$. Moreover,

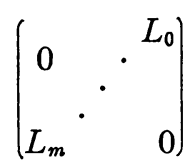

is the matrix of the symmetric bilinear function over $E_{\infty}^{2 k}$ relative to $\xi_{\infty}$. By Lemma 1 we have $\tau(E)=\tau_{\xi_{\infty}}\left(E_{\infty}\right)$. This concludes the proof of our theorem.

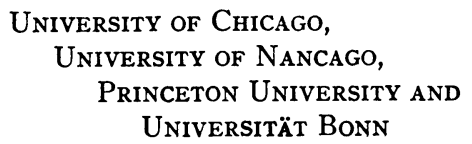

\title{
THE PERIPHERAL CHARACTER OF CENTRAL ELEMENTS OF A LATTICE ${ }^{1}$
}

\section{A. D. WALLACE}

A lattice being a Hausdorff space together with a pair of continuous lattice operations $(\wedge$ and $\vee$ ) the content of this note is best exhibited by quoting a corollary to our theorem: If a compact connected lattice is (topologically) situated in Euclidean n-space then its center is contained in its boundary. Thus, far from being "centrally located," the central elements are "peripheral."

The above is a consequence (see [3, p. 273]) of the

THEOREM. If $L$ is a compact connected lattice, if $R$ is an $(n, G)$-rim [3] for $L$ and if (i) $a$ is central [1, p. 27] or if (ii) $L$ is modular and $a$ is complemented then $a \in R$.

PROOF. The procedure is to introduce an appropriate multiplication into $L$ so that $L$ is a semigroup, to show that $L$ is not simple (in the semigroup sense [3]) and that $a$ is a left unit. Since $L$ is compact it has a zero and unit, 0 and 1 , as is well-known. Indeed, the set $\bigcap\{x \vee L \mid x \in L\}$ is easily seen to consist of exactly one element, namely 1 . If $a=1$ then the hypotheses of Theorem 1 of [3] are fulfilled using the multiplication $(x, y) \rightarrow x \wedge y$ so that 1 being a unit for the multiplication, $1 \in R$. If $a \neq 1$ let $x \cdot y=\left(a^{\prime} \wedge x\right) \vee y$, $a^{\prime}$ being a

Received by the editors September 15, 1956.

1 This work was supported by the National Science Foundation. 
complement of $a$, see $\S 9$, Chapter II of [1]. Under (i) or (ii) the multiplication is associative (loc. cit.) and is clearly continuous. It is immediate that $a \cdot y=y$ since $a^{\prime} \wedge a=0$, hence $a$ is a left unit. It is clear that $x \cdot 1=1$ so that $L \cdot 1 \cdot L=1 \cdot L$. If $1 \cdot L=L$ then 1 is a left unit for this multiplication by Theorem 1 of [2]. But $1 \cdot a=1 \neq a$.

Hence $L \cdot 1 \cdot L \neq L$ and it remains only to show that $x \cdot L=L$ implies $x \cdot R=R$, in order to verify the hypotheses of Theorem 1 of [3]. But $x \cdot L=\left\{\left(a^{\prime} \wedge x\right) \bigvee y \mid y \in L\right\}$ so that $x \cdot L=L$ gives $a^{\prime} \wedge x=0$ and hence $x \cdot R=\left\{\left(a^{\prime} \wedge x\right) \vee y \mid y \in R\right\}=\{y \mid y \in R\}=R$.

It is known (unpublished) that the center of any compact lattice is totally disconnected. L. W. Anderson (Tulane dissertation, 1956) has shown that a compact connected lattice which is (topologically) situated in the plane is distributive. In this case we see that all complemented elements are boundary-elements. It would be interesting to know if the Theorem remains valid upon the deletion (in (ii)) of the stipulation that $L$ be modular. The hypothesis of modularity was used only in obtaining the associativity of the multiplication.

REMARK. If one desires to forego the commutativity of addition and multiplication then a modular lattice may be regarded (in many ways) as a ring-like system. For $a \in L$ (modular!) let $x+y=(x \wedge a) \bigvee y$ and $x \cdot y=(x \bigvee a) \wedge y$. It is clear that addition and multiplication are idempotent and associative and that multiplication distributes over addition: indeed, $(x+y) z=y z=x z+y z$. Moreover $x+a=a=x a$ for all $x \in L$. If $L$ has a zero or unit or if $a$ has a complement then these elements play special roles, easily determined. Presumably the lattice structure can be recovered from such ring-like systems.

\section{REFERENCES}

1. G. Birkhoff, Lattice theory, New York, 1948.

2. A. D. Wallace, Cohomology, dimension and mobs, Summa Brasiliensis Mathematicae vol. 3 (1953) pp. 43-54.

3. - The Gebietstreue in semigroups, Neder. Akad. Wetensch. vol. 18 (1956) pp. 271-274.

TULANE UNIVERSITY 